\title{
Observation of Multiple Volume Reflection of Ultrarelativistic Protons by a Sequence of Several Bent Silicon Crystals
}

\author{
W. Scandale \\ CERN, European Organization for Nuclear Research, CH-1211 Geneva 23, Switzerland \\ A. Vomiero \\ INFM-CNR, Via Vallotti 9, 25133 Brescia, Italy \\ S. Baricordi, P. Dalpiaz, M. Fiorini, V. Guidi, and A. Mazzolari \\ INFN Sezione di Ferrara \& Università degli Studi di Ferrara, Dipartimento di Fisica, Via Saragat 1, 44100 Ferrara, Italy \\ G. Della Mea and R. Milan \\ INFN Laboratori Nazionali di Legnaro, Viale Università 2, 35020 Legnaro (PD) Italy \\ G. Ambrosi and P. Zuccon \\ INFN Sezione di Perugia, Via Pascoli, 06123 Perugia, Italy \\ B. Bertucci, W. Burger, and M. Duranti \\ INFN Sezione di Perugia \& Università degli Studi di Perugia, Dipartimento di Fisica, Via Pascoli, O6123 Perugia, Italy \\ G. Cavoto, R. Santacesaria, and P. Valente \\ INFN Sezione di Roma, Piazzale Aldo Moro 2, 00185 Roma, Italy \\ C. Luci \\ INFN Sezione di Roma \& Dipartimento di Fisica, Universita di Roma La Sapienza Piazzale A.Moro, 2 00185 Roma Italy \\ F. Iacoangeli \\ Dipartimento di Fisica, Universita di Roma La Sapienza Piazzale A.Moro, 200185 Roma Italy \\ E. Vallazza \\ INFN Sezione di Trieste, Via Valerio 2, 34127 Trieste, Italy \\ A. G. Afonin, Yu. A. Chesnokov, V. I. Kotov, V. A. Maisheev, and I. A. Yazynin \\ Institute of High Energy Physics, RU-142284 Protvino, Moscow Region, Russia \\ A. D. Kovalenko and A. M. Taratin \\ Joint Institute for Nuclear Research, Joliot-Curie 6, RU-141980 Dubna, Moscow Region, Russia
}

A. S. Denisov, Y. A. Gavrikov, Yu. M. Ivanov, L. P. Lapina, L. G. Malyarenko, V. V. Skorogobogatov,

V. M. Suvorov, and S. A. Vavilov

Petersburg Nuclear Physics Institute, 188300 Gatchina, Leningrad Region, Russia

D. Bolognini, S. Hasan, A. Mozzanica, and M. Prest

Università dell'Insubria, Via Valleggio 11, 22100 Como, Italy \& INFN Sezione di Milano Bicocca,

Piazza della Scienza 3, 20126 Milano, Italy

(Received 2 October 2008; published 23 February 2009)

The interactions of $400 \mathrm{GeV}$ protons with different sequences of bent silicon crystals have been investigated at the H8 beam line of the CERN Super Proton Synchrotron. The multiple volume reflection of the proton beam has been studied in detail on a five-crystal reflector measuring an angular beam deflection $\theta=52.96 \pm 0.14 \mu \mathrm{rad}$. The efficiency was found larger than $80 \%$ for an angular acceptance at the reflector entrance of $70 \mu \mathrm{rad}$, with a maximal efficiency value of $\varepsilon=0.90 \pm 0.01 \pm 0.03$.

DOI: 10.1103/PhysRevLett.102.084801

PACS numbers: 29.27.- a, 41.85.- $\mathrm{p}, 61.85 .+\mathrm{p}$

Directional effects arising in the interaction of charged particles with crystals have been investigated in the last decades and have been exploited for beam steering at different particle accelerators. Beam extraction or splitting 
by means of bent crystals is a well-established technique, conversely, crystal-assisted beam collimation is still under development even if the use of bent crystals in collimation systems was already proposed in the early nineties [1,2] for the new generations of high energy hadron colliders.

The basic idea is to use a bent crystal as an active primary collimator which deflects the beam halo particles at an optimal distance from the central beam orbit. For properly oriented crystals, coherent scattering from atomic planes replaces the scattering process of single atoms and beam halo particles can be deflected at larger angles and with a much reduced angular spread than in amorphous targets. This allows a more effective positioning of the subsequent absorbers and an overall increase of the collimation efficiency. Theoretical calculations and experimental campaigns have been carried out in the last decade [3-6] to develop the crystal assisted collimation with the main focus on channeling as the underlaying physical mechanism for deflection. These experiences pointed out the limits of this approach, arising both from the limited intrinsic channeling efficiency and its restricted angular acceptance. Multipass schemes [7] or the exploitation of a different deflection mechanism, the volume reflection, [8,9] have been proposed as viable solutions to optimize the collimation efficiency.

The charged particle volume reflection phenomenon in bent crystals predicted in $[10,11]$ and observed in $[12,13]$ has been studied at the CERN Super Proton Synchrotron (SPS) resulting in the deflection of a $400 \mathrm{GeV}$ proton beam of an angle of about $14 \mu \mathrm{rad}[9,14]$. The larger deflection efficiency and the wider angular acceptance of volume reflection (VR) with respect to channeling was firmly established. However, larger deflection angles would be desirable in the design of collimation systems. This can be achieved by means of multiple reflections through a sequence of crystals [15]: doubling of the deflection angle in a two crystals deflector system was recently demonstrated in [16].

In this Letter we report the observation of the multiple volume reflection (MVR) of $400 \mathrm{GeV} / c$ protons in a sequence of several bent crystals at the $\mathrm{H} 8$ beam line of the CERN SPS during the May and November 2007 run periods. The experimental layout shown in Fig. 1 corresponds to the May 2007 run and derives from the one described in [17] with improved tracking capabilities.

Crystals are mounted on mechanical holders fixed on a high-precision goniometric system which allows for an accurate positioning and orientation of the multicrystal deflector with respect to the beam. Two types of multicrystal deflectors were developed for this experiment to accommodate either five quasimosaic [18] or six to eight striplike silicon crystals [19]. A relative alignment between adjacent crystals with an accuracy much better than their bending angles (typically $\sim 100 \mu \mathrm{rad}$ ) is achieved in both deflectors types by means of an accurate mechanical assembly and the use of active controls. Both types of multicrystal deflectors were exposed to the proton beam and MVR was clearly observed with similar performance. We present here the detailed results obtained with quasimosaic crystals and a brief summary of striplike crystals performance.

The quasimosaic deflector made use of five crystal plates of $14 \times 10 \times 0.65 \mathrm{~mm}^{3}$ with (111) atomic planes disposed parallel to the $14 \times 0.65 \mathrm{~mm}^{2}$ face. Each crystal plate was bent on a cylinder with a radius of curvature $\sim 2.7 \mathrm{~m}$ by clamping between the concave and convex parts of the bending device to provide a $\sim 110 \mu \mathrm{rad}$ quasimosaic bend of the (111) planes. On the edge of the bending device, in the area where the proton beam should pass through the crystal, a special opening with a sizes of $5 \times$ $3 \mathrm{~mm}$, or $6 \times 1 \mathrm{~mm}$ for different samples was foreseen. Bending devices with clamped crystals were mounted on a support made from a metal plate with narrow deep cuts to provide the fine angular alignment of crystals using piezoelements put in these cuts.

High-precision tracking of individual proton trajectories before and after the interaction with crystals is performed by means of double-sided silicon microstrip detectors placed in pairs upstream (SD1,SD2) and downstream (SD3,SD4) the goniometer. The deflection angle is evaluated from the difference between the angular direction at incidence $\left(\theta_{\text {in }}\right)$, reconstructed with the SD1-SD2 detectors, and the angular direction after the crystal $\left(\theta_{\text {out }}\right)$, measured with SD2 and either of the SD3 or SD4 detectors. A systematic error of $3 \%$ in the evaluation of $\theta_{\text {out }}$ is introduced by the $0.3 \mathrm{~m}$ distance between the SD2 detector and the crystals and corrected on a track by track basis. The estimated resolution in the deflection angle measurement due to the SD spatial resolution is $3 \mu \mathrm{rad}$.

Data collection was organized in runs corresponding to different relative alignments of the crystals. In each run, an angular scan in the horizontal $(x z)$ plane was performed acquiring the events for different orientations of the goniometer with respect to the nominal beam direction.

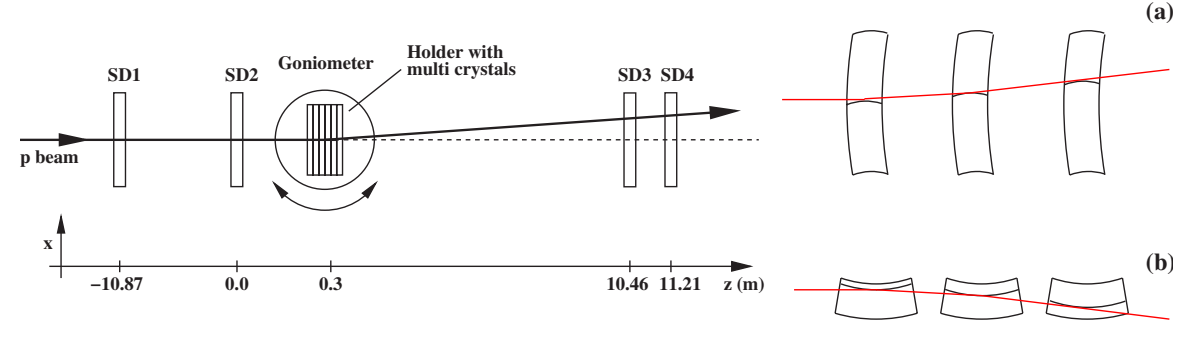

FIG. 1 (color online). Schematic layout of the experimental setup used to study multiple volume reflection at the H8 beam line of the CERN SPS. The trajectory of a particle undergoing a sequence of volume reflections in a series of aligned crystals is schematically represented on the right for quasimosaic (a) and strip crystals (b). 
The beam intensity distribution as a function of the track deflection angle (vertical axis) in different goniometer angular positions (horizontal axis) is shown in Fig. 2 as measured in the angular scans performed before (top) and after (bottom) the relative alignment of the five crystals.

Before the crystal alignment, the deflection effects arising from the proton interactions separately in each crystal are visible as peculiar structures in the track deflection angle distribution corresponding to five distinct goniometer orientation intervals. The channeling peak, at positive deflection angles of $\sim 100 \mu \mathrm{rad}$, and the VR region, for negative deflection angles of $\sim 10 \mu \mathrm{rad}$, are found in each interval for increasing values of the goniometer angle.

A Gaussian function has been used to describe the deflection angle distributions for volume reflected tracks in each crystal as in [14]. In Table I the mean deflection angles measured for the volume reflection in the five crystals are reported, as well as the corresponding goniometer positions.

The deflection angle and the efficiency for the fivefold volume reflection have been measured with the finely aligned crystal in the angular scan. In Fig. 2 (bottom) a
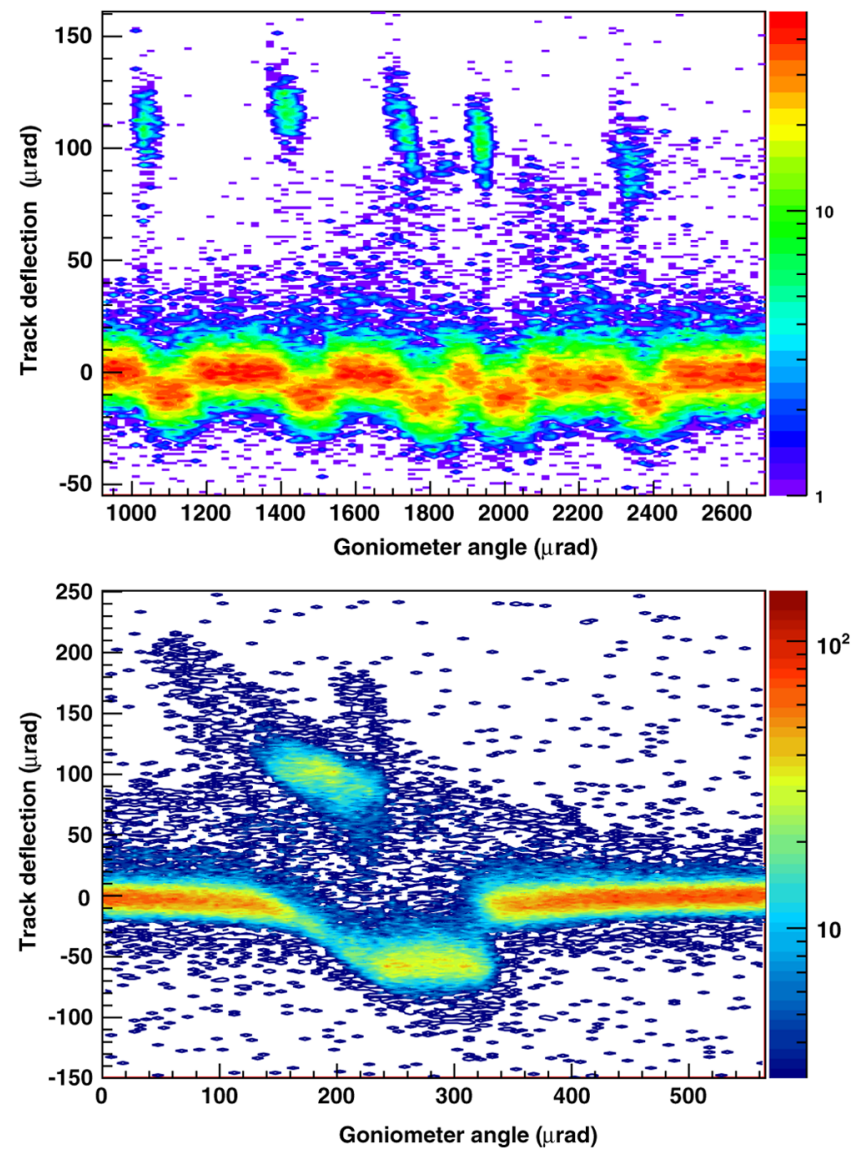

FIG. 2 (color online). Beam intensity distributions observed in the angular scans performed before (top) and after (bottom) the relative alignment of the five crystals. The beam intensity is reported as a function of the track deflection angle (vertical axis) in different goniometer angular positions (horizontal axis). net superposition of the volume reflection effect from the five crystals is clearly visible corresponding to a track deflection angle of $\sim 50 \mu \mathrm{rad}$ towards negative values. Conversely, deflection towards positive angles due to channeling is still of the same magnitude as in the unaligned crystals run, but the channeling peaks due to single crystals are merged for contiguous goniometer positions.

A Gaussian fit to the track deflection angle distributions was used to estimate the mean deflection angle $(\theta)$ and its dispersion $(\sigma)$ for channeled and volume reflected beam fractions as well as for the undeflected beam. The ratio between the number of events within $\pm 3 \sigma$ around the fitted peak value and the total number of collected events at each goniometer orientation defines the corresponding raw efficiency $\varepsilon_{R}$ for the beam component under study. The volume reflection efficiency is then obtained as $\varepsilon^{\mathrm{VR}}=$ $\varepsilon_{R}^{\mathrm{VR}} / \varepsilon_{R}^{U}$, where $\varepsilon_{R}^{U}=96.6 \pm 0.6 \%$ represents the raw efficiency in absence of deflection effects. The systematic uncertainties on the deflection angle are mainly related to the single Gaussian model used to fit the track deflection angle distributions. The sum of several Gaussian functions has been used to describe more in detail the deflection peaks but no systematic effect in the measured deflection angle has been found within the statistical accuracy. The systematic uncertainty on the efficiency measurement is mainly related to the criterion used to define the number of tracks associated to the fitted peak: multiple Gaussian fits and different widths of the association window were used to check the measurement stability and to estimate the systematic error in the efficiency value.

The results from the fit procedure are summarized in Fig. 3. The uppermost panel reports the raw efficiencies measured on channeled, volume reflected and unperturbed beam components as a function of the goniometer orientation. The corresponding fitted values of the mean deflection angle are shown in the bottom panel.

The transition towards the channeling effect is observed at the beginning of the angular scan: tracks are nearly undeflected up to a goniometer orientation $\alpha \sim 140 \mu \mathrm{rad}$, where a steep increase of the channeled beam component is observed. Two beam components can be distinguished in the subsequent steps of the angular scan: the channeled tracks, which dominate up to $\alpha=230 \mu \mathrm{rad}$, and the unchanneled beam fraction steadily deflected towards nega-

TABLE I. Mean deflection angles for volume reflected $\left(\theta_{\mathrm{VR}}\right)$ protons as measured with the five not aligned crystals. The corresponding goniometer position intervals $\left(\alpha_{\mathrm{VR}}\right)$ are also reported.

\begin{tabular}{lc}
\hline \hline$\alpha_{\mathrm{VR}}(\mu \mathrm{rad})$ & $\theta_{\mathrm{VR}}(\mu \mathrm{rad})$ \\
\hline$\alpha \in[1080,1100]$ & $9.6 \pm 0.3$ \\
$\alpha \in[1480,1500]$ & $9.6 \pm 0.3$ \\
$\alpha \in[1780,1800]$ & $12.5 \pm 0.4$ \\
$\alpha \in[2020,2040]$ & $10.2 \pm 0.4$ \\
$\alpha \in[2380,2400]$ & $11.8 \pm 0.4$ \\
\hline \hline
\end{tabular}



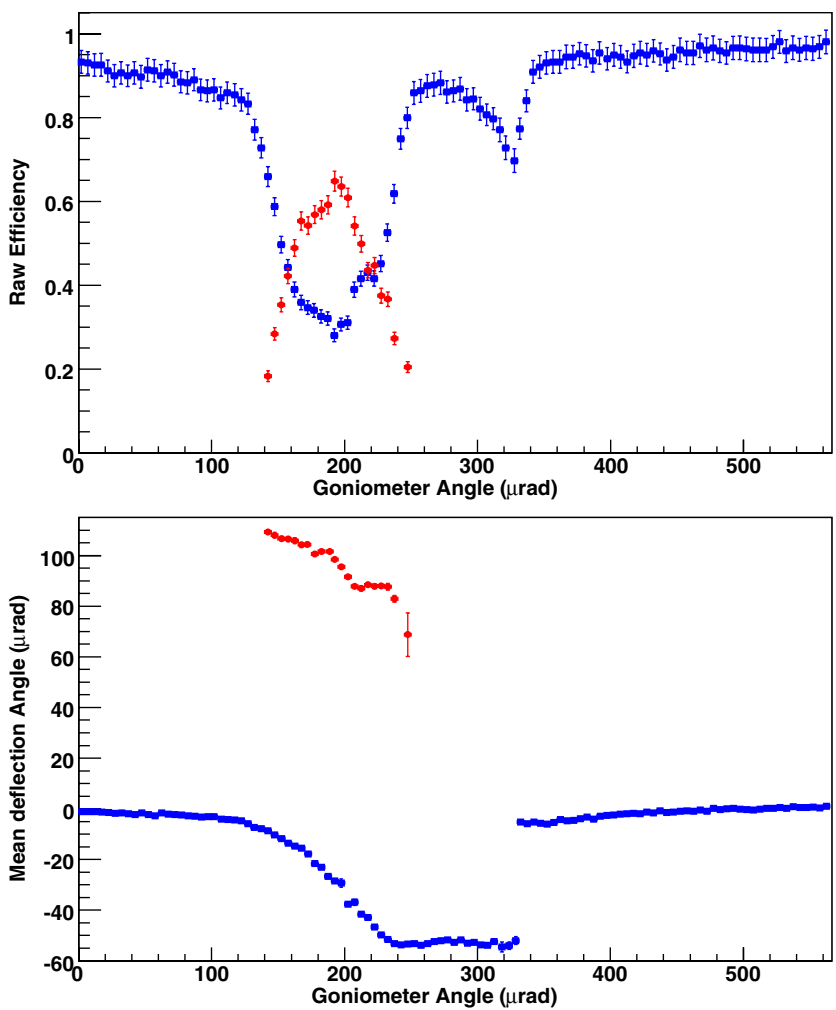

FIG. 3 (color online). Raw efficiency (top) and mean deflection angle (bottom) measured for channeled (red or gray) and volume reflected or undeflected tracks (blue or darkgray) as a function of the goniometer position. Data refer to the angular scan with finely aligned crystals.

tive angles. The onset of volume reflection is observed at goniometer orientations where the channeling effect comes to its end: a mean deflection angle $\theta=-52.96 \pm 0.14 \mu \mathrm{rad}$ is observed in a wide interval of the goniometer orientation $(245<\alpha<315 \mu \mathrm{rad})$ with a VR efficiency greater than $80 \%$. The measured deflection is consistent with the sequence of reflections in the five crystals as it can be estimated from the sum of the deflection angle values obtained for the single crystals $\theta_{1-5}=53.7 \pm 0.8 \mu \mathrm{rad}$ (Table I). The maximal volume reflection efficiency, $\varepsilon^{\mathrm{VR}}=0.90 \pm$ $0.01 \pm 0.03$, is recorded for $250<\alpha<290 \mu \mathrm{rad}$. The VR effect extinction appears for $315<\alpha<340 \mu \mathrm{rad}$ : a unique Gaussian peak is used to describe the deflection angle distributions in the subsequent scan steps. In the very last part of the angular scan, $\alpha>475 \mu \mathrm{rad}$, no deflection effects are observed: only events collected at these crystal orientations are used to study the unperturbed beam properties and to determine the $\varepsilon_{R}^{U}$ value.

The MVR effect was also measured with two different sequences of striplike crystals. In the eight strip deflector, the crystals had been bent along (111) crystallographic plane, with length and bending angle being equal to $2.25 \mathrm{~mm}$ and $300 \mu \mathrm{rad}$, respectively. The measured deflection angle of the volume reflected beam was $70 \pm$ $5 \mu \mathrm{rad}$ with an efficiency of $\varepsilon=0.85 \pm 0.05$. In the six strip deflector, the crystals had been bent along the (110) crystallographic plane, with length and bending angle values of $2 \mathrm{~mm}$ and $150 \mu \mathrm{rad}$ respectively. The measured deflection angle of the volume reflected beam was $40 \pm$ $1.5 \mu \mathrm{rad}$ with a reflection efficiency of $\varepsilon=0.93 \pm 0.04$.

In conclusion, this experiment has confirmed that multiple volume reflections of ultrarelativistic protons in sequences of bent crystals can be obtained with high efficiency over a wide entrance angular range. For the first time, the volume reflection effect has been successfully exploited in order to achieve a sizable angular deflection of ultrarelativistic protons. This result strengthens the possibility to use crystal reflectors for the beam collimation of LHC and encourages the further developments in this field which have been recently proposed [20].

We are grateful to Professor L. Lanceri (INFN \& University of Trieste) who provided the tracking detectors. This work was partly supported by Russian Foundation for Basic Research (Grant No. 06-02-16912),'Elementary Particle Physics and Fundamental Nuclear Physics" Program of Russian Academy of Sciences, INFN-NTAHCCC program, the INTAS-CERN Foundation (Grant No. 05-103-7525), and the MIUR 2006028442 project.

[1] M. Maslov, N. Mokhov, and I. Yazynin, SSCL Report No. 484, 1991.

[2] M. D. Bavizhev et al., in Proceedings of the 1991 PAC Conference (IEEE, New York, 1991), p. 177.

[3] D. Trbojevic et al., in Proceedings of the 1988 EPAC Conference (World Scientific, Singapore, 1989).

[4] R. P. Fillet et al., Phys. Rev. ST Accel. Beams 9, 013501 (2006).

[5] V. M. Biryukov, A. I. Drozhdin, and N. V. Mokhov, in Proceedings of the 1999 PAC Conference (IEEE, New York, 1999), p. 1234.

[6] A. G. Afonin et al., Phys. Rev. Lett. 87, 094802 (2001).

[7] V. M. Biryukov et al., Nucl. Instrum. Methods Phys. Res., Sect. B 234, 23 (2005).

[8] I. A. Yazynin, in Proceedings of the 1995 PAC Conference (IEEE, New York, 1995), p. 1952.

[9] W. Scandale et al., Phys. Rev. Lett. 98, 154801 (2007).

[10] A. M. Taratin and S. A. Vorobiev, Phys. Lett. A 119, 425 (1987).

[11] A. M. Taratin and S. A. Vorobiev, Nucl. Instrum. Methods Phys. Res., Sect. B 26, 512 (1987).

[12] Y. M. Ivanov et al., Phys. Rev. Lett. 97, 144801 (2006).

[13] Y. M. Ivanov et al., JETP Lett. 84, 372 (2006).

[14] W. Scandale et al., Phys. Rev. ST Accel. Beams 11, 063501 (2008).

[15] A. M. Taratin and W. Scandale, Nucl. Instrum. Methods Phys. Res., Sect. B 262, 340 (2007).

[16] W. Scandale et al., Phys. Lett. B 658, 109 (2008).

[17] W. Scandale et al., Rev. Sci. Instrum. 79, 023303 (2008).

[18] Y.M. Ivanov, A. A. Petrunin, and V. V. Skorobogatov, JETP Lett. 81, 99 (2005).

[19] A. G. Afonin et al., JETP Lett. 67, 781 (1998).

[20] W. Scandale and M. Prest, CERN Report No. CERNSPSC-2008-014/SPSC-P-335, 2008. 